\title{
Innovative Concept for a Heavy-Load Aircraft Utilizing a Two-Dimensional Wing
}

\author{
M. Leroy Spearman* \\ NASA Langley Research Center, Hampton, Virginia , 23681
}

\begin{abstract}
[Abstract] Heavy-load aircraft of conventional wing-body-tail design have become very large. Excessive size of such aircraft may present problems in the manufacturing process. In addition, large wing spans may cause some difficulties in ground handling. Increasing lift loads on large span cantilever wings will also increase the strength of the wing tip vortex. The concept presented herein proposes a means for substantially increasing the lift load capability of an aircraft without increasing the overall length and span of the configuration. The concept has a rectangular wing with a relatively low span and a large chord to provide the area required for high lift. Large fuselages are attached at each wing tip to provide the volume required for heavy loading. The fuselages serve as endplates for the wing and should preclude tip flow so that two-dimensional flow might be established on the wing. Elimination of the wing tip flow should prevent the formation of a tip vortex and eliminate the tip vortex hazard to trailing aircraft. Exploratory wind tunnel tests of such an aircraft concept have been conducted. Lessons learned from these tests are discussed herein in an effort to determine the validity of the concept.
\end{abstract}

\section{Introduction}

$\mathrm{O}$ ver the years there has been a progressive increase in the size of aircraft intended for transporting heavy loads. In general, the geometric arrangement of these aircraft has remained essentially unchanged with a single center body having a forward-mounted wing and an aft tail assembly. Increasing the load-carrying capacity of these aircraft has been accomplished by simply increasing the size of the configuration components. For example, this can be illustrated with the long line of Boeing transport airplanes. The Boeing 247 transport, developed in 1933, had a crew of 3 and carried 10 passengers and about 400 pounds of mail. The airplane was about 52 feet long, had a wing span of 74 feet, and a gross weight of 13,659 pounds. By comparison, the present day Boeing 747 carries about 470 passengers, is 250 feet long, has a wing span of 225 feet and gross weight of 970,000 pounds. In recent years there has been some speculation about developing an even larger transport aircraft that would have about twice the capacity of the current Boeing 747. Some concepts have been conceived in which the aircraft is simply a still further enlarged version of today's current aircraft. The extremely large size of such a design introduces some possible new concerns. Present manufacturing plants may need to be enlarged to facilitate the construction of such an aircraft. The attachment of conventional cantilevered wing panels to the fuselage may result in an increase in structural weight due to the material required to sustain the large bending and twisting loads that would result from the greater wing lift loads. The large lift loads that would be generated by very large aircraft would also result in an increase in the strength of the tip vortex that could affect trailing aircraft. In addition, extremely large physical size may lead to ground handling problems of an aircraft insofar as taxi-way clearance and gate-docking are concerned. With the possible new problem areas for very large aircraft in mind, the innovative aircraft concept proposed herein represents an attempt to eliminate or alleviate some of the problems.

\section{Discussion}

The impact of a number of fatal crashes of transport aircraft in the landing pattern was a factor leading to the proposed innovative design concept. Many of these fatal accidents were caused when an aircraft in the landing pattern was following too close behind a large aircraft and flew into a strong vortex flow that had been generated at the wing tip of the large aircraft. It was believed that the occurrence of such accidents would become even more likely with very large aircraft having greater wing spans and greater wing area. An initial thought was that such

*Distinguished Research Associate, AIAA Fellow 
accidents could be eliminated simply by eliminating the tip vortex. The installation of wing tip fences does, of course, alter the wing tip flow but the tip vortex formation is not completely eliminated. The concept proposed herein represents an attempt to completely eliminate flow around the wing tip by placing very large bodies at each wing tip. Thus the entire wing surface is located between the two large bodies and there are no outboard cantilevered wing panel. The large bodies are, in fact, twin fuselages that provide the required volume. The wing has a relatively low aspect ratio rectangular planform, a restricted span, and the required area is provided through the use of a large wing chord. The large fuselages attached to each tip of the wing provide the required volume. Thus, there is no free wing tip, spanwise flow should be eliminated, and tip vortex flow with the attendant induced drag should not exist. Wind tunnel tests at low speeds have been made of the proposed concept. Detailed results from these tests are presented in references 1 through 4 . The present paper presents a summary of what has been learned from the experimental results.

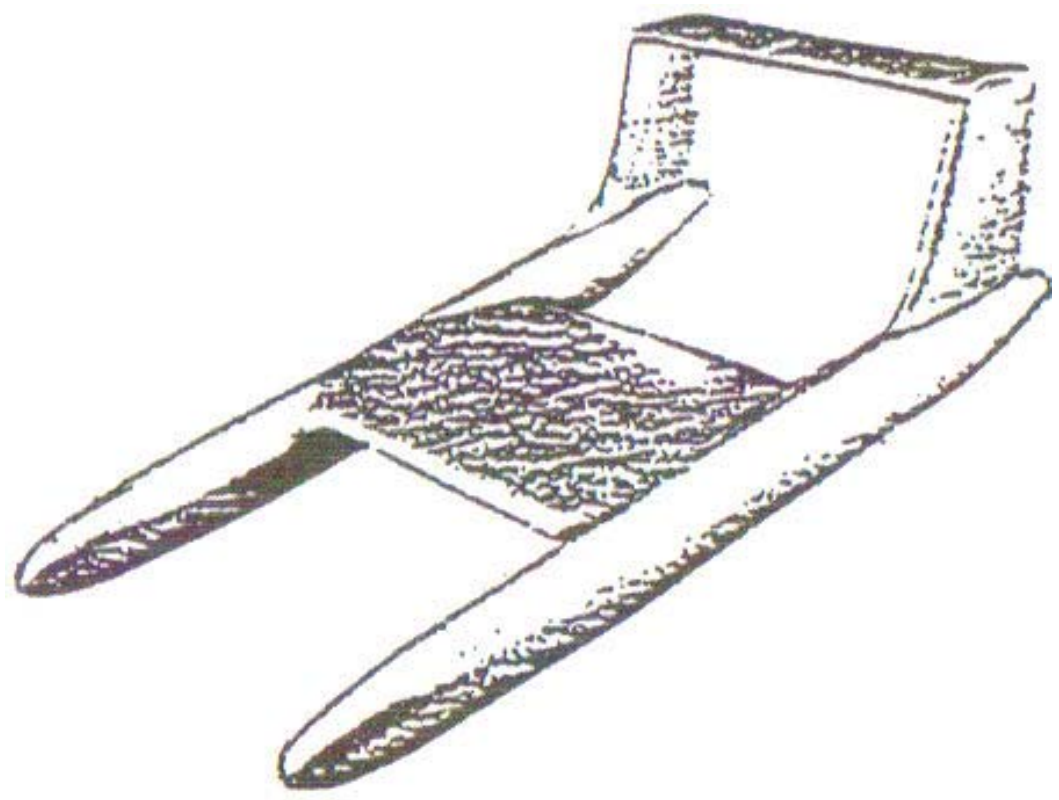

Oil flow tests were conducted so that the surface flow pattern might be made visible. For the plain wing, the flow pattern clearly showed the region of inward flow of air on the upper surface near the wing tip as the angle of attack was increased. This inward flow of air would result in some loss of wing lift and would also promote the formation of a vortical flow pattern aft of the wing tip. When the bodies were attached to the wingtips, however, the flow pattern remained streamwise across the entire span of the wing. Thus no loss of lift would occur at the wing tip and no vortical flow would be formed aft of the wing. Subsequently, pressure distribution measurements were made at several stations across the span of the wing. These measurements indicated that the wing section pressure distributions remained essentially constant across the span of the wing. Thus, from the oil flow tests and the pressure distribution tests it was determined that the span load distribution was rectangular, that no spanwise flow was apparent, and that two-dimensional flow existed on the wing. With two-dimensional flow it is likely that a laminar boundary would be established on the wing and a relatively low skin friction coefficient would be realized.

Force tests were made to determine the general aerodynamic stability and control characteristics. Component buildup tests were made for the wing alone, the wing with bodies, and the complete model with wing, body, vertical and horizontal tail. The addition of the bodies to the wing produced an increase in drag that would be expected due to the frontal area and skin friction of the bodies. The addition of the bodies to the wing did result in an increase in the lift-curve slope. This increase was believed to be due primarily to the elimination of the wing tip induced drag rather than from any lift generated by the bodies. The addition of the vertical tails produced a decrease in drag. This interesting result was caused by a favorable interference flow field acting on the vertical tail. Oil flow photographs showed that the flow around the body induced an inward flow of air at the vertical tail. This inward flow of air produced a small positive angle of attack on the vertical tail surface. As a result an inward sideforce vector was established at the vertical tail. This sideforce vector was inclined forward and provided a small thrust component that reduced the drag. The addition of the horizontal tail added a little drag but also added an increase in lift. Oil flow pictures indicated that the two-dimensional flow was established on the horizontal tail since the attachment to the tips of the vertical tails precluded any tip flow. 
The static longitudinal, lateral and directional stability characteristics were found to be satisfactory. No control deflection tests have been made but it is reasonable to believe that no difficulty would be encountered in achieving satisfactory control characteristics. Control surfaces could easily be placed on the horizontal tail for pitch control and on the vertical tails for directional control. Various types of flaps could be devised for the wing trailing edge that could provide for lift control and for roll control. Wing leading-edge flaps or extensions could also be employed. One force test was made with a full-span wing leading-edge extension. Although this resulted in a decrease in the wing aspect ratio, there was a measurable increase in the lift-curve slope. While this is contrary to the usual relation between aspect ratio and lift-curve slope, it is evident that two-dimensional flow exists on the wing.

Flow visualization tests were made with a grid and a pressure probe to survey the flow field wake aft of the model. With the plain wing the wake survey clearly showed the formation of a vortex aft of the wing tip. For the complete configuration, however, the flow field aft of the model was essentially clear of any vortex flow. This result has favorable implications for reducing the severity of incidents involving trailing aircraft encountering the wake of other aircraft. As noted earlier, it was the trailing vortex hazard that, in part, prompted the conceptual design of the innovative large aircraft utilizing a two-dimensional wing that is described herein.

Some thought has been given relevant to this concept when scaled to be about twice the size and capacity of a current large transport such as the Boeing 747. Such a concept would employ two B-747 fuselages and would have twice the wing area of a B-747. The overall length, of course, would be the same as a B-747. The overall width (span) that was considered was less than that for a B-747 and the large wing area required was achieved by using a large wing chord that would be possible with this type of arrangement. The chord length was estimated to be about 100 feet and with a 12 percent thick airfoil section the maximum wing thickness would be 12 feet. With this much thickness it was thought that submerged engines in the wing would be a possibility. Thus it was conjectured that a large load carrying aircraft could be conceived that, when compared to a B-747, would have twice the load capability, would have the same length with less span, and would have no trailing vortex.

Some thought has also been given to the development of a STOL version or a Wing-in-Ground Effect (WIG) version of the concept. Sitting on the surface, such a concept with two bodies, a high wing location, and with trailing edge flaps deflected would create an essentially sealed chamber beneath the wing. Jet engines mounted on the inboard side of the bodies forward of the wing could direct the jet flow into the chamber under the wing that would create a large positive pressure that would lift the vehicle vertically. Such an arrangement could provide for short takeoff with flight in free air as an aircraft or flight could continue as a wing-in-ground effect vehicle.

Closing Thoughts.- Some other factors have been considered relative to such an aircraft when compared to a conventional wing-body-tail design having the same capacity. The factory space required for the construction would be much less for the innovative design. The absence of large span cantilevered wing panels could have a significant effect on the structure and the weight factors. The wing arrangement for the proposed concept would avoid the structural and weight requirements associated with the bending, twisting and attachment of cantilevered wing panels. All things considered thus far, it appears that the innovative concept with the two-dimensional wing is a reasonable approach to a large aircraft having twice the load capacity of a present day jumbo jet but with no increase in length, a decrease in span and with no hazardous trailing vortex.

\section{References}

1. Spearman, M. Leroy: A High-Capacity Airplane Design Concept Having an Inboard-Wing Bounded by Twin Tip-Mounted Fuselages. AIAA 97-2276.

2. Spearman, M. Leroy; and Karen M. Feigh: An Airplane Configuration with an Inboard Wing Mounted Between Twin Fuselages. AIAA 98-0440.

3. Spearman, M. Leroy: An Airplane Design Having a Wing with a Fuselage Attached to Each Tip. AIAA 2001-0536.

4. Orr, M., Magill, S.A., Schetz, J.A., Marchman, J.F. III, Mason, W. H.,and Grossman, B.: Experimental Study of the Aerodynamic Properties of the Inboard Wing Concept AIAA 2001-0577.

5. Patterson, J. Claude, Jr, and Dhanvada, M. Rao. Exploratory Low-Speed Wind Tunnel Investigation of an Inboard-Wing Transport Aircraft Concept. ViGYAN Inc. Hampton, VA, March 18, 1997. 